\title{
Early results of the ongoing Polish Registry of Valve Thrombosis after Transcatheter Aortic Valve Implantation (ZAK-POLTAVI)
}

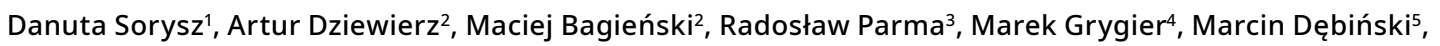 \\ Piotr Kübler ${ }^{6}$, Maciej Stąpór7, Dariusz Jagielak ${ }^{8}$, Kleczyński Paweł², Stanisław Bartuś2 , Dariusz Dudek ${ }^{1,2}$ \\ 1 Department of Cardiology and Cardiovascular Interventions, University Hospital, Jagiellonian University Medical College, Kraków, Poland \\ 2 2nd Department of Cardiology, Institute of Cardiology, Jagiellonian University Medical College, Kraków, Poland \\ 3 3rd Department of Cardiology, Medical University of Silesia, Katowice, Poland \\ 4 1st Department of Cardiology, Poznan University of Medical Sciences, Poznań, Poland \\ 5 1st Department of Cardiac Surgery, American Heart of Poland, Bielsko-Biała, Poland \\ 6 Department of Heart Diseases, Wroclaw Medical University, Wrocław, Poland \\ 7 Department of Interventional Cardiology, John Paul II Hospital, Kraków, Poland \\ 8 Department of Cardiac and Vascular Surgery, Medical University of Gdańsk, Gdańsk, Poland
}

\section{KEY WORDS}

aortic stenosis, transcatheter aortic valve implantation, valve thrombosis
Correspondence to: Danuta Sorysz, MD, PhD, 2nd Department of Cardiology and Cardiovascular Interventions, University Hospital, Jagiellonian University Medical College, ul. Jakubowskiego 2 , 30-688 Kraków, Poland, phone: +48124002250 , email: dtsorysz@op.pl Received: January 26, 2020 Revision accepted: June 6, 2020 Published online: June 8, 2020. Kardiol Pol. 2020; 78 (7-8): 681-687 doi:10.33963/KP.15426 Copyright by the Author(s), 2020

\section{ABSTRACT}

BACKGROUND Conflicting data exist regarding the risk factors for transcatheter heart valve thrombosis (THVT). In addition, no optimal pharmacological strategy to treat THVT has been established so far.

AIMS The aim of this study was to assess the incidence, risk factors, diagnostic workup, and treatment of THVT in Poland.

METHODS Data were collected retrospectively in the multicenter registry of patients with THVT (ZAK-POLTAVI) between November 2008 and November 2018. Transcatheter heart valve thrombosis was defined as an increased mean transvalvular gradient accompanied by a decreased effective orifice area or severe aortic regurgitation, reversible after treatment. Baseline characteristics and procedural data were compared between patients with THVT and those without THVT (matched by age, sex, and diabetic status).

RESULTS In a group of 2307 patients undergoing transcatheter aortic valve implantation (TAVI), 26 patients with THVT were identified (incidence, 1.14\%). In half of the patients, THVT was diagnosed within 6 months after TAVI. As compared with the control group, patients with THVT more frequently had chronic obstructive pulmonary disease $(P=0.035)$, a smaller aortic valve area $(P=0.007)$, a higher mean postprocedural transvalvular gradient $(P=0.037)$, and a lower platelet count $(P=0.029)$ at the time of the diagnosis. A total of 24 patients $(84.6 \%$ ) received anticoagulation therapy for THVT, and complete resolution of THVT was noted in 12 individuals (46.1\%). We observed thromboembolic complications in 2 patients (7.7\%). CONCLUSIONS Transcatheter heart valve thrombosis is a rare complication of TAVI. However, a higher risk of THVT may be expected in patients with chronic obstructive pulmonary disease, a smaller aortic valve area, a higher mean postprocedural transvalvular gradient, and a lower platelet count. Anticoagulation alone or combined with antiplatelet therapy seems to be the optimal pharmacological treatment in this population.

INTRODUCTION Transcatheter aortic valve implantation (TAVI) is a well-established treatment method for severe aortic stenosis in elderly patients who are ineligible for surgery or at high risk of surgery. Transcatheter aortic valve implantation may also be considered in intermediate-risk patients with favorable anatomy. ${ }^{1,2}$ However, there is an increasing awareness of possible transcatheter heart valve thrombosis (THVT), and this complication seems to be a relevant limitation of the current TAVI techniques. Importantly, a common risk factor for 


\section{WHAT'S NEW?}

There is an increasing awareness of possible transcatheter heart valve thrombosis (THVT). However, data regarding the detection of this condition in everyday practice are limited. In this article, we reported data on THVT in Poland. The incidence of THVT in the study patients treated with transcatheter aortic valve implantation was $1.14 \%$. The risk factors for THVT included chronic obstructive pulmonary disease, a smaller aortic valve area, a higher mean postprocedural transvalvular gradient, and a lower platelet count. The majority of patients received anticoagulation as the first-line treatment for THVT, and complete resolution of THVT was observed in half of the patients. In 2 patients, thromboembolic complications of THVT were confirmed. Nonetheless, in some patients, discontinuation of anticoagulation may be the reason for THVT recurrence. Further studies are needed to define the optimal pharmacological treatment to prevent and treat THVT.

THVT is the absence of anticoagulation. ${ }^{3-7}$ On the other hand, prolonged and aggressive antiplatelet and / or antithrombotic therapy after TAVI may increase the risk of bleeding in elderly patients with multiple comorbidities. The majority of data on THVT come from large studies with close follow-up, including the analysis of advanced imaging and serial multislice computed tomography (MSCT) scans. Data regarding the detection of THVT in everyday practice are limited. Thus, we sought to assess the incidence, diagnostic workup, treatment, and outcomes of THVT in daily clinical practice.

METHODS In the Polish Registry of Valve Thrombosis after Transcatheter Aortic Valve Implantation (Polish, Polski Rejestr Zakrzepicy Przezskórnie Wszczepianej Zastawki Aortalnej [ZAK-POLTAVI]), 6 centers were asked to fill in a questionnaire regarding periprocedural and follow-up data of TAVI procedures conducted between November 2008 and November 2018. Data were collected retrospectively. Transcatheter heart valve thrombosis was defined as an increased mean transvalvular gradient accompanied by a decreased effective orifice area (EOA), reversible after treatment. Alternatively, it was regarded as an inexplicable, early rise of the mean gradient (eg, with no signs of endocarditis or improvement of left ventricular stroke volume) or new, reversible after treatment, severe aortic regurgitation. ${ }^{8}$ Transthoracic echocardiography (TTE) was used as a standard, first-line diagnostic tool and, in the majority of centers, TTE findings were verified using transesophageal echocardiography (TEE) or MSCT. Standard post-TAVI treatment differed among the 6 centers, but included single antiplatelet therapy with aspirin $(75 \mathrm{mg} / \mathrm{d})$ or dual antiplatelet therapy (DAPT) with aspirin $(75 \mathrm{mg} / \mathrm{d})$ and clopidogrel ( $75 \mathrm{mg} / \mathrm{d})$ for 6 months, followed by lifelong aspirin therapy $(75 \mathrm{mg} / \mathrm{d})$. In patients with indications for oral anticoagulants (OACs), the decision as to whether treatment with vitamin $\mathrm{K}$ antagonists (VKAs) / non-vitamin $\mathrm{K}$ antagonist oral anticoagulants (NOACs) with or without a single antiplatelet agent should be initiated was left at the discretion of the treating physician. The platelet count was evaluated at the time of diagnosis of THVT. In the control group, the last available data were used.

Statistical analysis Data were analyzed according to the established standards of descriptive statistics. Results were presented as the number (percentage) of patients or median (interquartile range [IQR]). The control group included 26 patients without THVT, matched by age, sex, and diabetic status. Patients were selected from the database of TAVI procedures performed in the Institute of Cardiology, Kraków, Poland, during the study period. Differences between groups were assessed using the $\mathrm{X}^{2}$ test and the Fisher exact test for dichotomous variables and the Mann-Whitney test for continuous variables. All tests were 2 -sided and a $P$ value less than 0.05 was considered significant. All statistical analyses were performed using the Statistica 13.3 software (Tibco Software, Inc., Palo Alto, California, United States).

RESULTS Between November 2008 and November 2018, 2307 TAVI procedures were performed and 328 Edwards XT and 260 Edwards S3 (Edwards Lifesciences, Irvine, California, United States), 489 CoreValve, 826 Evolut R, 107 Evolut Pro, 3 Engager (Medtronic Scientific, Minneapolis, Minnesota, United States), 119 Lotus (Boston Scientific, Marlborough, Massachusetts, United States), 17 Jena Valve (JenaValve Technology, GmbH, Munich, Germany), 85 Acurate Neo (Boston Scientific, Marlborough, Massachusetts, United States), 43 Portico (St. Jude Medical, Minneapolis, Minnesota, United States), and 30 NVT (NVT, GmbH, Hechingen, Germany) valves were implanted. During follow-up, 26 patients with THVT were identified (the study group) based on an increased transvalvular gradient. Thus, the estimated incidence of THVT was low, of about $1.14 \%$ (0.3\% for Edwards XT, 2.7\% for Edwards S3, 0.8\% for CoreValve / Evolut R, 5.9\% for Lotus, and 2.3\% for Portico valves).

The median (IQR) age of patients with THVT was 81.5 (76-86) years, and 12 patients were female (66.7\%) (TABLE 1). The increased transvalvular gradient was confirmed on echocardiography performed at the median (IQR) time of 268 (176401) days after TAVI. Thrombosis was observed in 13 patients (50\%) within 6 months. In 11 patients (42.3\%), THVT was confirmed later than at 12 months after TAVI. Early thrombosis (developed earlier than at 3 months) was noted only in 2 patients (7.7\%). We recorded a significant rise of the transvalvular gradient with a median (IQR) 
TABLE 1 Baseline characteristics along with echocardiographic and procedural data of the study patients

Variable

Patients with valve thrombosis $(n=26) \quad$ Matched controls $(n=26) \quad P$ value

Age, $y$

Female sex

$82(77-86)$

BMI, $\mathrm{kg} / \mathrm{m}^{2}$

eGFR, $\mathrm{ml} / \mathrm{min} / 1.73 \mathrm{~m}^{2}$

$18(69.2)$

Platelets, $\times 10^{3} / \mu \mathrm{l}$

Platelets $<150 \times 10^{3} / \mu \mathrm{l}$

NYHA class III or IV

$25.5(23.9-30.6)$

$60(50-70)$

163 (151-209)

6 (23.1)

Arterial hypertension

16 (61.5)

Diabetes

23 (88.5)

Atrial fibrillation

9 (34.6)

$8(30.8)$

Previous MI

Previous PCI

8 (30.8)

Previous CABG

12 (46.2)

$3(11.5)$

COPD

8 (30.8)

Previous stroke/TIA

3 (11.5)

Pacemaker

Logistic EuroSCORE I, \%

0

STS score, \%

Baseline AV transvalvular gradient, $\mathrm{mm} \mathrm{Hg}$, mean (range)

Baseline $\mathrm{AV}$ area, $\mathrm{cm}^{2}$

Baseline LVEF, \%

Baseline SPAP, mm Hg

Baseline aortic regurgitation grade 3 or 4

Transfemoral access

Device implanted

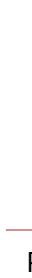

\begin{tabular}{ll}
\hline \multicolumn{1}{l}{ Edwards Sapien } \\
\hline Lotus \\
\hline Jena \\
\hline Portico \\
\hline Prosthesis size & $23 \mathrm{~mm}$ \\
\hline $25 \mathrm{~mm}$ \\
\hline $26 \mathrm{~mm}$ \\
\hline $27 \mathrm{~mm}$ \\
\hline $29 \mathrm{~mm}$ \\
\hline $31 \mathrm{~mm}$ \\
\hline
\end{tabular}

\begin{tabular}{ll} 
& $4(19.2)$ \\
\hline CoreValve and / or Evolut R & $25(96.2)$ \\
\hline
\end{tabular}

$11.4(8.9-16.5)$
$4.1(3.4-6.9)$

7 (26.9)

0

$56.5(37-74)$

$0.5(0.4-0.8)$

$52.5(40-60)$

$81.5(75-84)$ 0.61

18 (69.2)

0.99

$28.3(26.6-32.4) \quad 0.14$

$47(37-67) \quad 0.08$

$211.5(173-235)$

0.029

2 (7.7)

0.25

20 (76.9)

0.23

25 (96.2) 0.61

9 (34.6)

0.99

9 (34.6)

0.77

$10(38.5)$

0.56

$8(30.8)$

0.25

4 (15.4)

0.99

2 (7.7)

0.035

1 (3.8)

0.61

1 (3.8)

0.67

$18.6(9-24.8)$

0.11

4.8 (3.6-8.1)

0.53

$50(40-60)$

0.49

$0.7(0.6-0.9)$

0.007

$60(40-65)$

0.32

$48(41-68)$

0.15

0
0.05

21 (80.8)

0.05

$8(30.8)$

0.19

14 (53.8)

2 (11.5)

1 (3.8)

1 (3.8)

0

$10(38.5)$

7 (26.9)

0.74

1 (3.8)

1 (3.8)

7 (26.9)

9 (34.6)

$2(7.7)$

0

$5(19.2)$

$8(30.8)$

1 (3.8)

$1(3.8)$

$1(3.8)$

Maximal postprocedural AV transvalvular gradient, $\mathrm{mm} \mathrm{Hg}$

Postprocedural AV transvalvular gradient, $\mathrm{mm} \mathrm{Hg}$, mean (range)

Postprocedural LVEF, \%

Postprocedural aortic regurgitation grade 3 or 4

$24(20-30)$

14 (9.5-18)

16.5 (12-24)

0.005

$50(45-55)$

$9.5(6-13)$

0.012

$50(35-60)$

0.83

0

0

0.99

Data are presented as the number (percentage) of patients or median (interquartile range) unless otherwise indicated.

Abbreviations: AV, aortic valve; BMI, body mass index; CABG, coronary artery bypass grafting; COPD, chronic obstructive pulmonary disease; eGFR, estimated glomerular filtration rate; LVEF, left ventricular ejection fraction; MI, myocardial infarction; NYHA, New York Heart Association; PCI, percutaneous coronary intervention; SPAP, systolic pulmonary artery pressure; STS, Society of Thoracic Surgeons; TIA, transient ischemic attack 
maximal transvalvular gradient of 44.5 (3272) $\mathrm{mm} \mathrm{Hg}$ and a mean (minimum-maximum) gradient of 23 (19-41) mm Hg. Additionally, a decrease in the median (IQR) EOA from $1(1-2) \mathrm{cm}^{2}$ after the procedure to $1(1-1) \mathrm{cm}^{2}$ at follow-up was observed. The majority of patients demonstrated dyspnea exacerbation (13 patients [72.2\%]). In our study group, TEE was performed in 8 patients (30.8\%), MSCT in a single patient (3.8\%), both modalities were used in 11 patients (42.3\%), and none of them in 6 patients (23.1\%). At the time of THVT diagnosis, patients were still on DAPT (12 patients [46.2\%]) or aspirin (7 patients [26.9\%]). Seven patients (26.9\%) were treated with OACs, including 3 individuals (11.5\%) receiving the combination of a NOAC and a single antiplatelet agent.

As compared with the control group, patients with THVT more frequently had chronic obstructive pulmonary disease $(P=0.035)$, a smaller aortic valve area (AVA) $(P=0.007)$, and a higher mean postprocedural transvalvular gradient $(P$ $=0.037$ ). There was no difference between groups in the percentage of patients with a platelet count below $150 \times 10^{3} / \mu \mathrm{l}$ (TABLE 1). The platelet count was lower in the THVT group (median [IQR], 163 [151-209] vs 211.5 [173-235]; $P=0.029$ ).

A large variability in subsequent treatment was observed. For the treatment of THVT, 24 patients $(84.6 \%)$ received anticoagulation, including 8 patients (30.8\%) on anticoagulation alone, 6 (23.1\%) on anticoagulation and aspirin, $8(30.8 \%)$ on anticoagulation and clopidogrel, and $2(7.7 \%)$ on anticoagulation and DAPT. In 2 patients (7.7\%), current treatment with aspirin alone (1 case) and DAPT (1 case) was maintained. Importantly, in 3 patients (11.5\%) already receiving VKAs, the target international normalized ratio was raised from 2.5 to 3 . Complete resolution of THVT was observed in $12 \mathrm{pa}$ tients (46.1\%). Only a slight improvement was confirmed in 8 patients (30.8\%), and no change of the gradient was seen in 6 patients $(23.1 \%$; including 4 treatment-naive patients). Embolic events were noted in 2 patients $(7.7 \%)$ - central retinal artery embolization and ischemic stroke in each case separately-interestingly, just after starting anticoagulation. In a single patient, THVT recurred after transient discontinuation of warfarin, despite the use of enoxaparin as a bridge to noncardiac surgery. A patient with atrial fibrillation underwent left atrial appendage closure with the Watchman device and required blood transfusion because of gastrointestinal bleeding. In that patient, a significant increase of the transvalvular gradient was observed following anticoagulation withdrawal.

DISCUSSION Transcatheter heart valve thrombosis is a rare yet potentially dangerous complication that may affect various types of valves used for TAVI. Importantly, this condition carries an increased risk of embolic events and may impact valve durability. On the other hand, THVT is not always symptomatic. Thus, its detection may require more attention during follow-up. The observed incidence of $1.14 \%$ was low yet comparable to that reported in a study by Latib et $\mathrm{al}^{9}$ who reported the incidence of $0.6 \%$ when only TTE was used for detection of THVT. Importantly, conventional post-TAVI TTE follow-up is the most frequent method for valve assessment, which is inferior to contrast-enhanced MSCT for the detection of THVT. Using MSCT, 2 various types of THVT can be determined: hypoattenuated leaflet thickening and hypoattenuation affecting motion. ${ }^{10}$ Thus, diagnosis based on MSCT increased the sensitivity for THVT detection to up to $6.1 \%$ to $14.3 \%$. Transthoracic echocardiography enables clinicians to diagnose the rise of the transvalvular gradient due to reduced leaflet motion (the stage of hypoattenuation affecting motion). Hypoattenuated leaflet thickening is difficult or even impossible to visualize using TTE and it is also referred to as subclinical valve thrombosis. However, the assessment of the thickness of the transcatheter heart valve leaflet and its mobility by TEE is most likely improved compared with MSCT, owing to the superior temporal resolution of TEE. 10,11

In addition, as heterogenous criteria for the diagnosis of THVT exist, the described frequency may also be affected by the definition adopted..$^{8-13}$ For example, the Valve Academic Research Consortium-2 defines structural valve deterioration as valve-related dysfunction (mean aortic gradient $>20 \mathrm{~mm} \mathrm{Hg}$, EOA $<0.9-1.1 \mathrm{~cm}^{2}$, and / or dimensionless valve index $<0.35$, and / or moderate or severe prosthetic valve regurgitation) or need for a repeat procedure (TAVI or surgical aortic valve replacement). ${ }^{12}$ Lancellotti et $\mathrm{al}^{14}$ suggested incorporating an increase in the mean gradient during stress echocardiography at follow-up to the criteria of possible obstruction (mean transvalvular gradient, 10-19 $\mathrm{mm} \mathrm{Hg}$ ) and significant obstruction (mean transvalvular gradient $>20 \mathrm{~mm} \mathrm{Hg}$ ). In our study, we used the definition proposed by Pislaru et $\mathrm{al},{ }^{8}$ with an additional criterion of the increase in the transvalvular gradient larger than $50 \%$ of the baseline value (in the absence of increased stroke volume), particularly useful in the low-flow, low-gradient conditions. Of note, in selected cases, the rise of the transvalvular gradient can be a result of left ventricular ejection fraction improvement after TAVI.

Importantly, risk factors for THVT have not been defined yet. For instance, conflicting data regarding the impact of sex and valve size on the risk of THVT exist. $7,9,13,14$ In our small group of patients with THVT, most subjects were female and had small valves. In addition, a higher transvalvular postprocedural gradient and smaller preprocedural AVA were observed in 
the THVT group. Therefore, potentially, a more calcified valve with a smaller AVA and possible underexpansion of the implanted valve (with a higher postprocedural gradient) might be a risk factor for THVT. On the contrary, a larger size of the implanted valve was suggested to be associated with the slow flow (especially in the base of the sinus of Valsalva) and a higher risk of THVT. ${ }^{7}$ In addition, the valve-in-valve procedure was also found to be a risk factor for THVT. ${ }^{7,15}$ Interestingly, the higher transvalvular gradient may correspond with elevated shear stress. This may cause platelet activation and enhance the prothrombotic state. ${ }^{16}$ On the other hand, shear stress may play a role in bleeding complications by shedding of the platelet receptor and loss of high-molecular-weight multimers of von Willebrand factor. ${ }^{16,17}$ Other authors suggested that there may be an interaction between high shear stress and platelet activation by a potential platelet disruption effect due to a high transvalvular gradient. ${ }^{17,18}$ The observed platelet count was lower in the THVT group compared with the control group, and no difference was observed in the rate of thrombocytopenia. Although a cause-effect relationship cannot be ruled out, the lower platelet count in the THVT group might be related to antithrombotic treatment initiated to manage this complication and / or differences in the timing of platelet count assessment between groups. A temporal drop of the platelet count is a known phenomenon following bioprosthesis implantation, associated with unfavorable outcomes after TAVI. ${ }^{19}$

Other risk factors for THVT include nonuse of post-TAVI VKA treatment or allergy to any stent material. The majority of the study patients were observed in the last 2 years when newly developed valve types were used. New technologies reduce the risk of paravalvular leaks using additional materials (eg, skirt) to seal the space between the valve stent and calcifications of the leaflets and the annulus. However, such materials, long scaffolds in particular, might incite thrombus formation. Other theories have suggested that the prothrombotic effect of a metal stent and the importance of neosinus formation with turbulent flow. ${ }^{20}$ Transcatheter heart valve thrombosis is more frequent in patients treated with balloon-expandable valves. ${ }^{7}$ Interestingly, in our study group, THVT was also more common in patients with chronic obstructive pulmonary disease and those with a lower glomerular filtration rate. Thus, the general inflammatory status may play a crucial role in the development
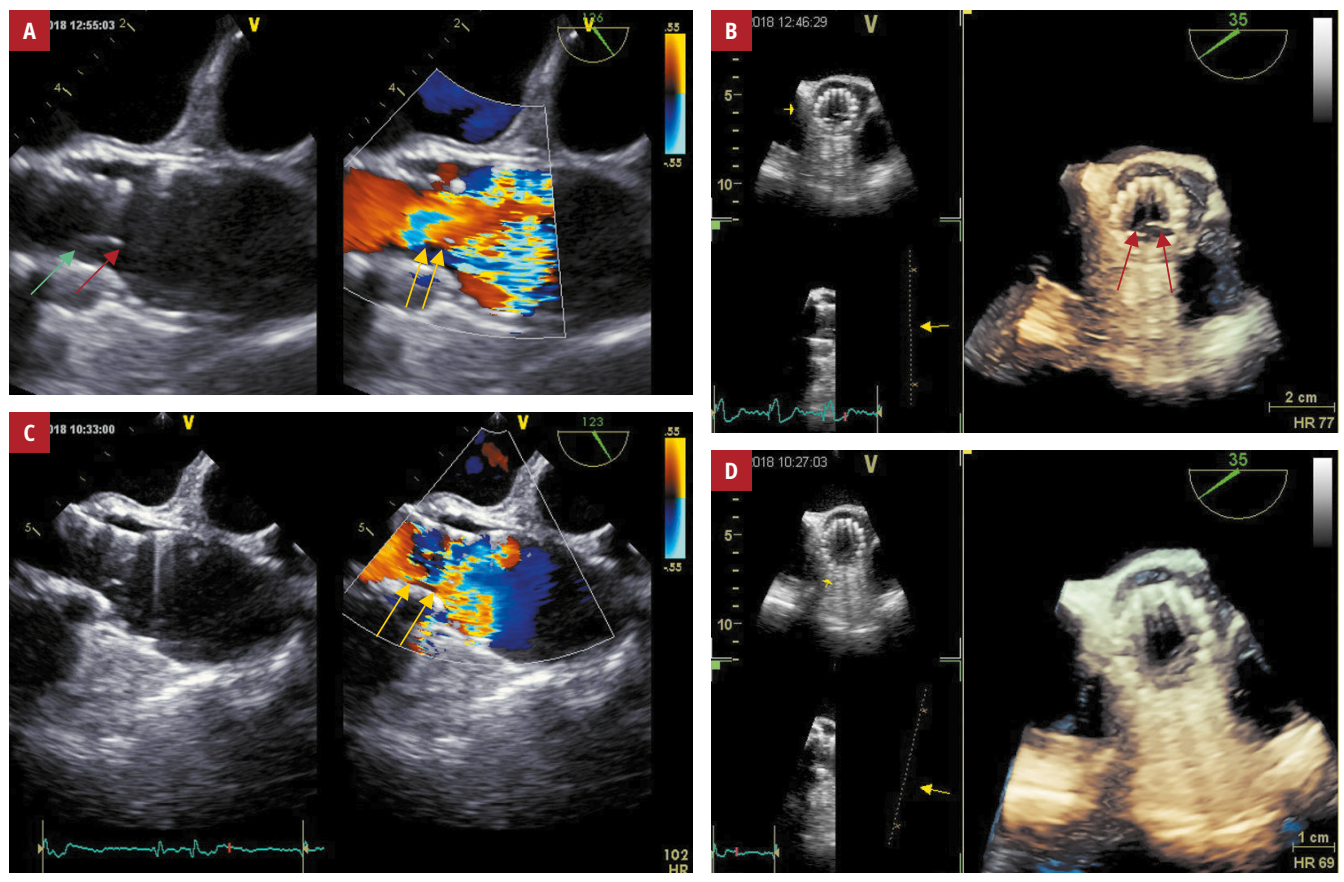

FIGURE 1 Illustrative case 1. At routine follow-up 6 months after Evolut R Pro valve implantation, an 83-year-old man reported no symptoms, but a slight increase of the transvalvular gradient on transthoracic echocardiography and reduced leaflet motion on transesophageal echocardiography (TEE) were observed. The patient showed good response to vitamin $\mathrm{K}$ antagonist and clopidogrel treatment, but recurrence was noted after discontinuation of vitamin K antagonist therapy. A - long-axis TEE view of the valve placed during the transcatheter aortic valve implantation (TAVI) procedure: 2 -dimensional (left) and color Doppler (right) imaging at the same projection showing limited leaflet motion (red arrow) with reduced flow (yellow arrows) and the thrombus (green arrow); B - 3-dimensional TEE reconstruction of the TAVI valve demonstrating reduced mobility of the leaflet (red arrows); C - long-axis TEE view of the TAVI valve: 2-dimensional (left) and color Doppler imaging (right) at the same projection showing larger flow through the valve (yellow arrows); D - 3-dimensional TEE reconstruction showing normal mobility of the leaflets 

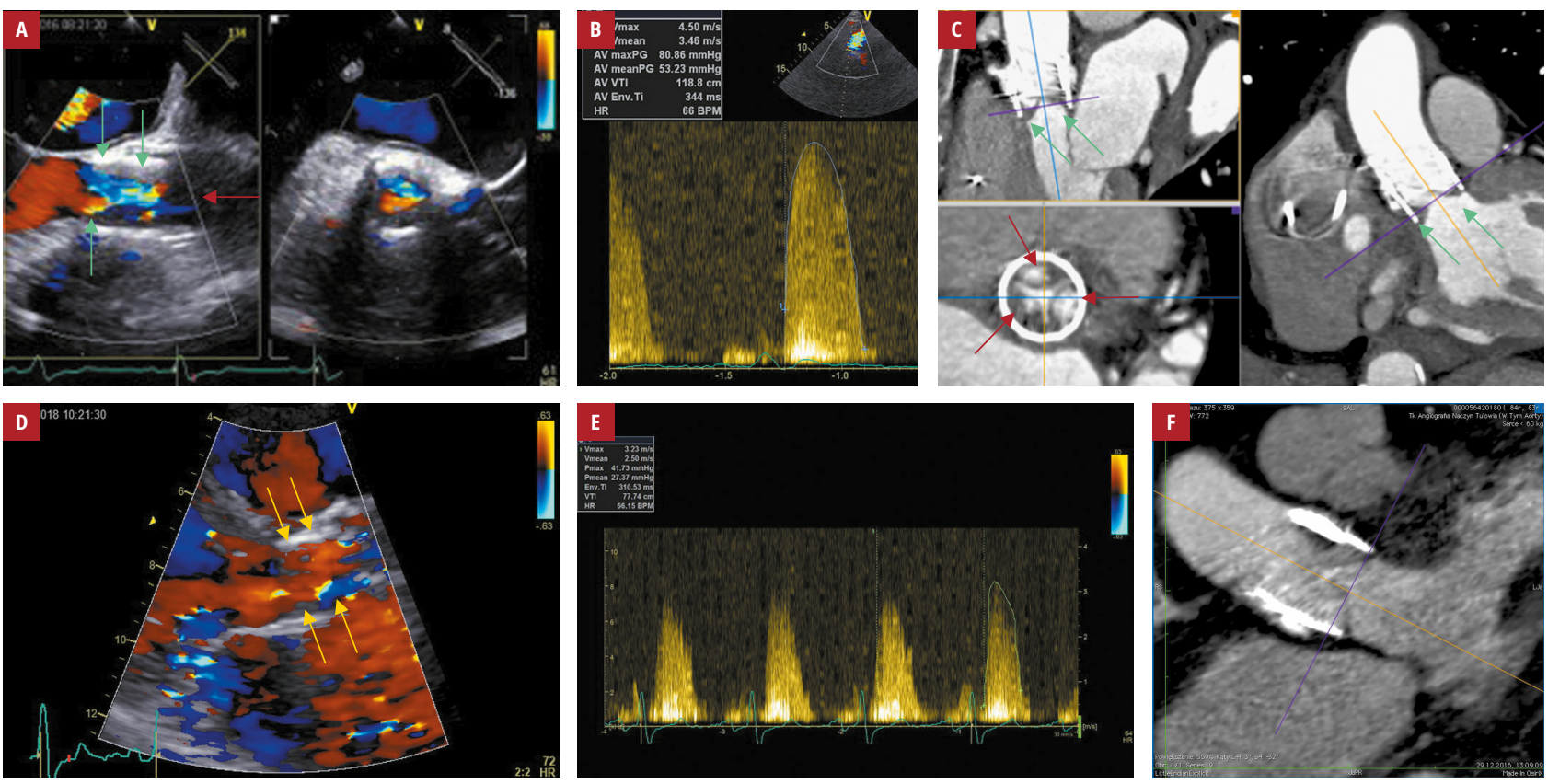

FIGURE 2 Illustrative case 2. At follow-up at 6 months after Lotus 23 valve implantation, a 82-year-old woman presented with symptoms typical of New York Heart Association class III/ IV, gradually worsening starting from 2 months after the procedure. Treatment with a reduced dose of enoxaparin was delayed because of transient ophthalmologic contraindications. Complete symptom resolution was not achieved, but relief and a reduced gradient during treatment were observed. A-C - Lotus valve thrombosis: A - 2-dimensional (left) and color Doppler (right) long-axis transesophageal echocardiography (TEE) view of the transcatheter aortic valve, showing a decreased leaflet opening (red arrow) and a thrombus (green arrows); B - right parasternal TTE view: a continuous-wave (CW) aortic transvalvular gradient of 81/53 mm Hg; C - computed tomography angiography showing a thrombus (green arrows) on the Lotus valve and reduced leaflet motion (red arrows). D-F - TEE after pharmacological treatment: D - long-axis parasternal color Doppler TTE view demonstrating larger flow through the valve (yellow arrows); E - right parasternal TTE view showing a reduced CW transvalvular gradient of up to $42 / 27 \mathrm{~mm} \mathrm{Hg} ; \mathbf{F}$ - computed tomography angiography demonstrating a considerable improvement of thrombosis

of THVT in patients after TAVI. Interestingly, a higher body mass index was suggested to have a protective effect in patients undergoing TAVI, ${ }^{21}$ being numerically higher in patients without THVT. We cannot exclude the impact of the body mass index, as well as other body composition parameters, on the risk of THVT. Further larger studies are needed to address this issue.

According to recent guidelines, surgical, noncritical valve thrombosis should be treated with VKA and aspirin. ${ }^{2}$ In line with the previous studies, anticoagulation combined with VKA was effective in most of our study patients and an increase of the mean EOA after treatment was confirmed (FIGURE 1). We did not observe any significant drop in the transvalvular gradient, possibly because of lack of VKA treatment in 2 patients and the delayed treatment start in a single patient (FIGURE2). The observed recurrence of THVT after discontinuation of warfarin may indicate that short-term warfarin treatment may be insufficient. ${ }^{13}$ Also, a limited treatment effect was observed in a patient with delayed treatment initiation, which may indicate the significance of early THVT detection and initiation of anticoagulation to prevent deterioration of the valve function. We observed 2 patients with obstructive THVT without amelioration despite the initiation of anticoagulation but with satisfactory clinical outcomes. Other authors also reported spontaneous resolution of THVT. ${ }^{6}$ This phenomenon may explain the discrepancy between the incidence of incidental THVT and clinically overt, obstructive THVT. A single patient required surgery and postoperative complications resulted in death. Most importantly, THVT carries an increased risk of embolic events and may affect valve durability. ${ }^{20,11}$ In our study group, a single case of central retinal artery embolization and another case of ischemic stroke were observed.

Limitations Our study was limited by retrospective data collection and unavailability of MSCT scans to confirm the diagnosis in the majority of patients. Identification of THVT predictors was not possible due to incomplete clinical data for all patients without this complication, treated during the study period. To address this limitation, we compared baseline characteristics and procedural data of patients with THVT with matched controls without THVT. However, the data of matched controls were retrieved only from a single center. The majority of THVT cases were identified in the final part of the enrollment period. It may indicate an increased awareness and the learning curve for detection of THVT using TEE and MSCT. 
Conclusions In conclusion, THVT is a rare complication of TAVI and may be observed after implantation of various types of valves. A higher risk of THVT may be expected in patients with chronic obstructive pulmonary disease, a smaller AVA, a higher mean postprocedural transvalvular gradient, and a lower platelet count. The optimal antiplatelet and antithrombotic therapy to prevent and treat this complication still needs to be investigated, although OACs with or without antiplatelet therapy seem to be effective. comparison among conventional, stentless, rapid-deployment, and transcatheter valves. Int J Cardiol. 2019; 296: 43-50.

18 Miceli A, Gilmanov D, Murzi M, et al. Evaluation of platelet count after isolated biological aortic valve replacement with Freedom Solo bioprosthesis. Eur J Cardiothorac Surg. 2012; 41: 69-73.

19 Kalińczuk $\measuredangle$, Zieliński K, Chmielak Z, et al. Effect on mortality of thrombo-inflammatory response after transcatheter aortic valve implantation. Am J Cardiol. 2019; D 124: 1741-1747.

20 Ranssinghe MP, Karlheninz P, McFadyen JD. Thromboembolic and bleeding complications in transcatheter aortic valve implantation: insights on mechanisms, prophylaxis and therapy. J Clin Med. 2019; 8: 280.

21 Tokarek TA, Dziewierz A, Sorysz D, et al. The obesity paradox in patients undergoing transcatheter aortic valve implantation: is there any effect of body mass index on survival? Kardiol Pol. 2019; 77: 190-197.

\section{ARTICLE INFORMATION}

CONFLICT OF INTEREST None declared.

OPEN ACCESS This is an Open Access article distributed under the terms of the Creative Commons Attribution-NonCommercial-NoDerivatives 4.0 International License (CC BY-NC-ND 4.0), allowing third parties to download articles and share them with others, provided the original work is properly cited, not changed in any way, distributed under the same license, and used for noncommercial purposes only. For commercial use, please contact the journal office at kardiologiapolska@ptkardio.pl.

HOW TO CITE SoryszD, DziewierzA, Bagieński M, et al. Early results of the ongoing Polish Registry of Valve Thrombosis after Transcatheter Aortic Valve Implantation (ZAK-POLTAVI). Kardiol Pol. 2020; 78: 681-687. doi:10.33963/KP.15426

\section{REFERENCES}

1 Leon MB, Smith CR, Mack M, et al. Transcatheter aortic-valve implantation for aortic stenosis in patients who cannot undergo surgery. N Engl J Med. 2010; 363: 1597-1607.

2 Baumgartner H, Falk V, Bax JJ, et al. 2017 ESC/EACTS Guidelines for the management of valvular heart disease. Eur Heart J. 2017; 38: 2739-2791.

3 De Marchena E, Mesa J, Pomenti S, et al. Thrombus formation following transcatheter aortic valve replacement. JACC Cardiovasc Interv. 2015; 8: 728-739.

4 Córdoba-Soriano JG, Puri R, Amat-Santos I, et al. Valve thrombosis following transcatheter aortic valve implantation: a systematic review. Rev Esp Cardiol. 2015; 68: 198-204.

5 Mylotte $D$, Andalib A, Thériault-Lauzier $P$, et al. Transcatheter heart valve failure: a systematic review. Eur Heart J. 2015; 36: 1306-1327.

6 Hansson NC, Grove EL, Andersen HR, et al. Transcatheter aortic valve thrombosis: incidence, predisposing factors, and clinical implications. J Am Coll Cardiol. 2016; 68: 2059-2069.

7 D'Ascenzo F, Salizzoni S, Saglietto A, et al. Incidence, predictors and cerebrovascular consequences of leaflet thrombosis after transcatheter aortic valve implantation: a systematic review and meta-analysis. Eur J Cardiothorac Surg. 2019; 56: 488-494.

8 Pislaru SV, Hussain I, Pellikka PA, et al. Misconceptions, diagnostic challenges and treatment opportunities in bioprosthetic valve thrombosis: lessons from a case series. Eur J Cardiothorac Surg. 2015; 47: 725-732.

9 Latib A, Naganuma T, Abdel-Wahab M, et al. Treatment and clinical outcomes of transcatheter heart valve thrombosis. Circ Cardiovasc Interv. 2015; 8: e00177910.

10 Yanagisawa R, Hayashida K, Yamada Y, et al. Incidence, predictors, and mid-term outcomes of possible leaflet thrombosis after TAVR. J Am Coll Cardiol Img. 2017; 10: 1-11.

11 Rosseel L, De Backer 0,Søndergaard L. Clinical valve thrombosis and subclinical leaflet thrombosis following transcatheter aortic valve replacement: is there a need for a patient-tailored antithrombotic therapy? Front Cardiovasc Med. 2019; 18: 44.

12 Kappetein AP, Head SJ, Généreux $P$, et al. Updated standardized endpoint definitions for transcatheter aortic valve implantation: the Valve Academic Research Consortium-2 consensus document. Eur Heart J. 2012; 33: 2403-2418.

13 Yanagisawa R, Hayashida K, Yamada Y, et al. Incidence, predictors, and mid-term outcomes of possible leaflet thrombosis after TAVR. JACC Cardiovasc Imaging. 2017; 10: 1-11.

14 Lancellotti $P$, Pibarot $P$, Chambers J, et al. Recommendations for the imaging assessment of prosthetic heart valves: a report from the European Association of Cardiovascular Imaging endorsed by the Chinese Society of Echocardiography, the Inter-American Society of Echocardiography, and the Brazilian Department of Cardiovascular Imaging. Eur Heart J Cardiovasc Imaging. 2016; 17: 589-590.

15 Scisło $\mathrm{P}$, Grodecki K, Bińczak D, et al. Valve-in-valve treatment of dysfunctional aortic bioprostheses - single-centre experience. Adv Interv Cardiol. 2018; 14: $425-428$.

16 Chen Z, Mondal NK, Ding J, et al. Paradoxical effect of nonphysiological shear stress on platelets and von Willebrand factor. Artif Organs. 2016; 40: 659-668.

17 Jiritano F, Santarpino G, Serraino GF, et al. Peri-procedural thrombocytopenia after aortic bioprosthesis implant: a systematic review and meta-analysis 